\title{
Radiation Data Acquisition with Research Stratospheric Balloon Incorporating IoT Telemetry System
}

\author{
Jiri Skorepa \\ Pavel Kovar \\ Pavel Puricer \\ Faculty of Electrical Engineering \\ Czech Technical University in Prague \\ Technicka 1902/2 \\ 16627 Praha
}

\begin{abstract}
The paper presents a radiation data measured in the atmosphere during the flight of a stratospheric balloon. The balloon carries the third version of the gondola with custom-developed electronic device integrating GPS, IoT, data storage and gamma radiation sensors. The radiation is measured by a miniature low-power silicon detector equipped with the plastic scintillator. The gondola enables to track the balloon during the whole stratospheric flight. The IoT network simplifies reception of the telemetry data on the ground.
\end{abstract}

Keywords: stratospheric balloon; gamma radiation; dosimeter; GPS tracking; Internet of Things;SigFox

\section{Introduction}

A helium-filled stratospheric balloon can carry the scientific equipment to the elevation of several tens of kilometres above the ground level. Hence, when carrying a battery-powered measurement device, the stratospheric balloons are well-suited for studying a variety of phenomena occurring in the Earth's atmosphere (Matsuzaka etal., 2000; Lokoshchenko \& Shifrin, 2009; Nishimura et al., 1978; Tobin et al., 2017;C. Mertens, 2016; C. J. Mertens et al., n.d.; Pfotzer, 1972). In other cases, the stratospheric balloons can be used as a cost-effective option to perform functionality tests of a device under development in the conditions which are difficult or expensive to simulate on the ground level (Laifr \& Kovar, 2018; Laporte, Evrard, \& Laurens,2006). Our work contributes to both cases since the custom device was developed toperform gamma radiation dose rates measurements in the atmosphere.

\section{Mission Descriptions}

In this paper, we use an unmanned stratospheric balloon equipped with gamma radiation detectors to measure the dose rate in the atmosphere during the balloon's flight at high altitudes. Those measurement data are sampled by a microcontroller and stored on the SD card and retrieved after landing. Due to weather effects, the unmanned balloon's trajectory and the landing point are unpredictable. Thus, an appropriate tracking system is needed. For this purpose, the telemetry system consisting of the Global Positioning System (GPS) receiver, microcontroller and SigFoxend point node is carried by the balloon together with the measurement equipment. Localization data provided by the GPS receiver are forwarded by the microcontroller to the SigFox node transmitting the data to the SigFox internet of things (IoT) network. The location of the balloon is then accessible in real-time, allowing us to track the balloon comfortably. The localization data are also synchronized to the data from performed dose rates measurements.

\section{Deployed Equipment}

For this measurement mission, the custom battery-powered gondola that integrates gamma radiation sensor, IoT communication modem, GPS receiver for telemetry and storage has been developed as shown in the Fig. 1. 


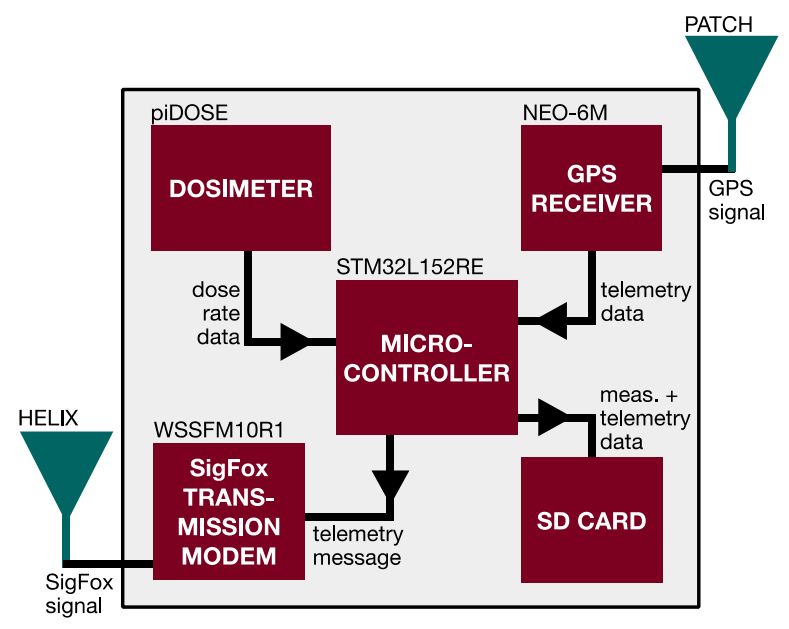

Figure 1: Block scheme of the developed measurement equipment carried by the balloon.

\subsection{Radiation Measurement System}

The gamma radiation dose rates measurement system is based on piDOSE DigitalCubeSat Dosimeter (SkyFox Labs, n.d.) used in (Laifr \& Kovar, 2018). Inside the dosimeter, there is X100-7 THD photodiode sensor (First Sensor, n.d.). In order to maximize the detected energy, a thallium-doped caesium iodide scintillation crystal is mounted on the top of each photodiode active area. When gamma rays are absorbed, the scintillation crystal responses with a maximal emission of radiation at $550 \mathrm{~nm}$. The size of the crystal is $4 \times 8 \times 8 \mathrm{~mm}$.

\subsection{Telemetry System}

The telemetry system includes two key components. The first one is the GPS receiver module u-blox NEO-6M (u-blox, n.d.) with simple patch antenna oriented upwards providing localization data. Those data of longitude, latitude and altitude are processed by a microcontroller STM32L152RE (STMicroelectronics, n.d.) into a word inNMEA RMC (National Marine Electronics Association Recommended Minimum sentence) format (Standard for Interfacing Marine Electronic Devices, 2008), assigned tothe current dose rate measurement data and stored on the SD card. From the same localization data, a message is then constructed and forwarded to the communication module SigFox node WSSFM10R1 (Sigfox, n.d.) with the short helix antenna operating in broadside mode and pointed to the Earth's surface. Then, the narrowband signal is transmitted in the $868 \mathrm{MHz}$ Short-Range Device (SRD) band and receivedby multiple SigFox gateways at the Earth's surface belonging to the SigFox IoT network. The gateway is connected to the internet via standard Transmission Control Protocol/Internet Protocol (TCP/IP) protocol, and all the data are forwarded to the SigFox network backend databases, in which the data are assigned to the account with the associated endpoint node module. The telemetry data from the tracked balloon are then accessible via an ordinary internet browser, from which they can be downloaded and processed, showing us a real-time location of the balloon. Such a solution was considered as significantly more comfortable comparing to the previously deployed UHF communication solution in (Puricer, Laifr, Kovar, \& Mikes, 2018) due to extensive SigFox IoT network coverage. Moreover, thanks to lower transmitted power of the SigFox endpoint node modem comparing to the previously used UHF communication modem in (Puricer et al., 2018), improved electromagnetic compatibility within the whole system was achieved. That led to less noisy data from thepiDOSE dose rate measurement sensor.

\section{Measurement Results}

The flight track of the released stratospheric balloon obtained by real-time receptionof telemetry data from SigFox communication can be found in the Fig. 2.

The ascent time took 83 minutes, whereas the descent time was 190 minutes giving 273 minutes in total as visible in the Fig. 3 together with radiation absorbed dose rates measured by the detector of the onboard dosimeter. According to onboard GPS receiver, the maximum altitude reached by the balloon was $22.12 \mathrm{~km}$, which is lowerthan the typical maximum altitude achievable by stratospheric balloon in general.

The vertical profile of the gamma radiation dosimetric quantities is shown in the Fig. 4. Despite the low reached altitude, the Regener-Pfotzer maximum (Carlson\& Watson, 2014; Reitz, 1993) can be distinguished at the altitude of $19 \mathrm{~km}$ with the dose rate value of $3.93 \mathrm{uGy} / \mathrm{h}$. 
Comparing to previous campaign taken in (Laifr \& Kovar, 2018), better electromagnetic compatibility of the deployed measurement system have been achieved. That resulted in less noisy measured radiation data. The descent altitude for the last balloon experiment was several kilometres lower than the descent altitude of the previous experiments. The problem could be a defect in the latex-made envelope of the balloon or improper gas filling of the balloon or other phenomena.

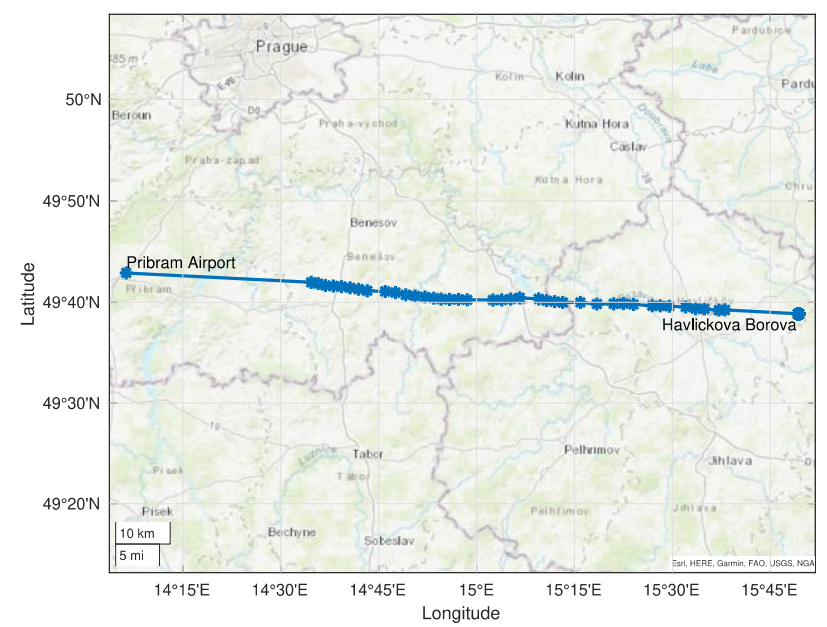

Figure 2: Real-time tracking of the balloon via SigFox communication from the starting location (Pribram Airport) to landing location (Havlickova Borova).

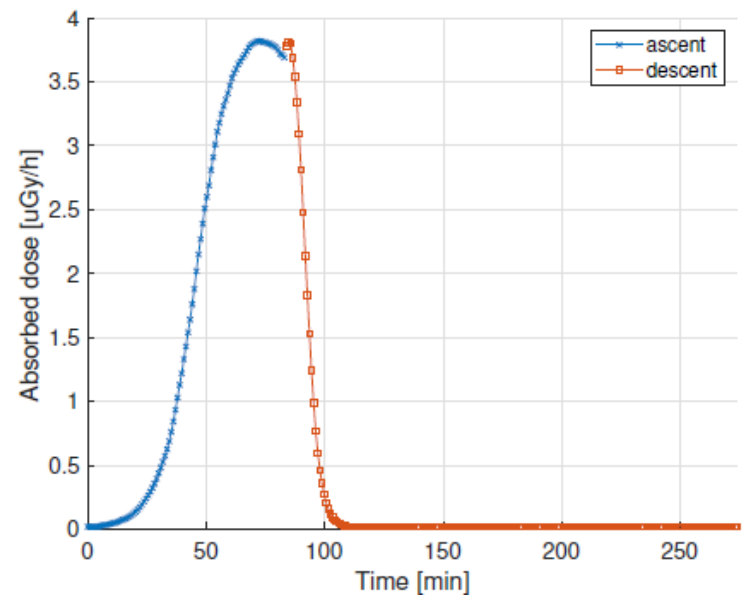

Figure 3: Measured gamma radiation dose rate related to the flight time.

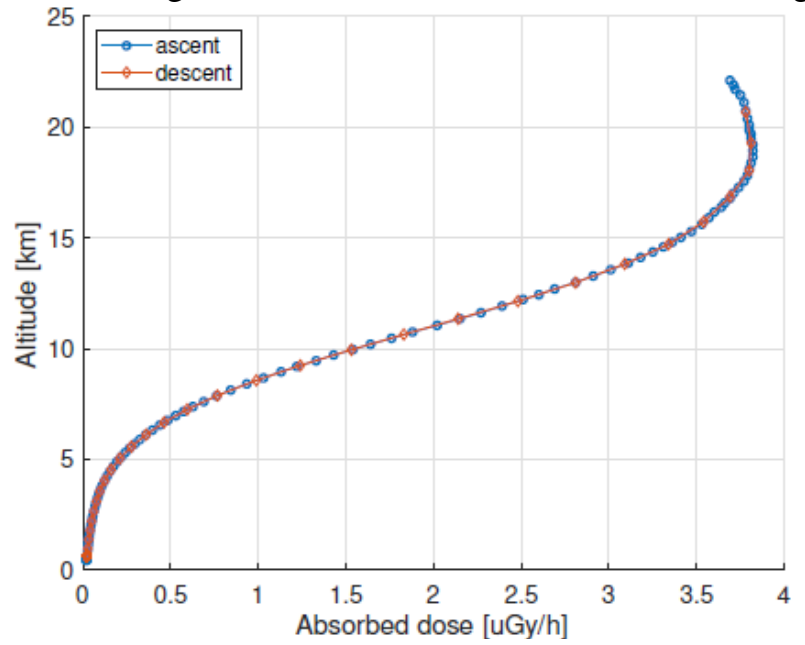

Figure 4: Altitude profile of absorbed gamma radiation dose rates measured by the onboard dosimeter. 


\section{Conclusions}

In this paper, the gamma radiation measurement system carried by the stratospheric balloon was constructed. The previously-tested radiation detector was used. Incorporating the SigFox communication modem, the balloon could have been conveniently tracked and retrieved. The Regener-Pfotzer maximum was reached and successfully measured.

\section{References}

Carlson, P., \& Watson, A. A. (2014). Erich Regener and the ionisation maxi-mum of the atmosphere. History of Geoand Space Sciences, 5(2), 175-182.Retrieved from https://www.hist-geo-space-sci.net/5/175/2014/ doi:10.5194/hgss-5-175-2014

First Sensor. (n.d.). PIN PD Data Sheet Part Description X100-7 THD. Retrieved from https://www.firstsensor.com/cms/upload/datasheets/X100-7_THD_3001447_3001448.pdf

Laifr, J., \& Kovar, P. (2018). High altitude baloon test fight of miniature low powerdosimeter for small satellites. Slaboproudyobzor, 74(1).

Laporte, P., Evrard, J., \& Laurens, A. (2006). Testing the first gamma-ray lens ona stratospheric balloon. In P. Ballmoos (Ed.), Focusing telescopes in nuclearastrophysics. Springer, Dordrecht. doi: 10.1007/978-1-40205304-7-44

Lokoshchenko, M., \&Shifrin, D. (2009, 02). Temperature stratification and altitude ozone variability in the low troposphere from acoustic and balloonsounding. Russian Meteorology and Hydrology, 34 , 72-82. doi: $10.3103 / \mathrm{S} 1068373909020022$

Matsuzaka, Y., Yamagami, T., Namiki, M., Toriumi, M., Ohta, S., Makino, F., \&Hirosawa, H. (2000, 12). Thin-film balloon for high altitude observation. Advancesin Space Research, 26, 1365-1368. doi: 10.1016/S02731177(00)00062-4

Mertens, C. $(2016,10)$. Overview of the radiation dosimetry experiment (rad-x) flightmission: Rad-x overview. Space Weather, 14. doi: 10.1002/2016SW001399

Mertens, C. J., Gronoff, G. P., Norman, R. B., Hayes, B. M., Lusby, T. C., Straume,T., . . Xu, X. (n.d.). Cosmic radiation dose measurements from the rad-x flightcampaign. Space Weather, 14 (10), 874-898. doi: 10.1002/2016SW001407

Nishimura, J., Fujii, M., Tawara, Y., Oda, M., Ogawara, Y., Yamagami, T., . .Yoshimori, M. (1978, March). Gammaray burst observed at balloon altitude., 272 , 337. doi: 10.1038/272337a0

Pfotzer, G. (1972). History of the use of balloons in scientific experiments. SpaceScience Reviews, 13 , 199-242. doi: $10.1007 /$ bf00175313

Puricer, P., Laifr, J., Kovar, P., \& Mikes, J. (2018). Telemetry system of FIK III.Stratospheric Balloon. In 2018 28th international conference radioelektronika(radioelektronika) (p. 1-4).

Reitz, G. (1993, 05). Radiation Environment in the Stratosphere. Radiation Protection Dosimetry, 48 (1), 5-20. Retrieved from https://doi.org/10.1093/oxfordjournals.rpd.a081837 doi: 10.1093/oxfordjournals.rpd.a0818376

Sigfox. (n.d.). Sigfox Communication Module WSSFM10R1. Retrieved from https://partners.sigfox.com/products/sfm10r1 (Accessed: 04/23/2020)

SkyFoxLabs. (n.d.). piDOSE-DCD Digital CubeSat Dosimeter. Retrieved fromhttp://www.skyfoxlabs.com/product/16cubesat-geiger-counter (Accessed: 04/23/2020)

Standard for Interfacing Marine Electronic Devices (Standard No. NMEA 0183 V4.00). (2008). National Marine Electronics Association.

STMicroelectronics. (n.d.). STM32L152RE - Ultra-low-power 32-bit MCU ARM R-based Cortex R-M3 with 512KB Flash, 80KB SRAM, 16KB EEPROM, LCD,USB, ADC, DAC. Retrieved from https://www.st.com/resource/en/datasheet/stm321152re.pdf (Accessed: 04/23/2020)

Tobin, R. J., Burr, A. W., Gilman, S. W., Hainsworth, W. J., Howell, A. S., \&Martin, K. M. (2017). Measuring radiation in the stratosphere: A high altitudeballoon journey. In Aiaa balloon systems conference. Retrieved from https://arc.aiaa.org/doi/abs/10.2514/6.2017-3788 doi: 10.2514/6.2017-3788

u-blox. (n.d.). NEO-6 GPS Modules. Retrieved from https://www.u-blox.com/en/product/neo-6-series (Accessed: $04 / 23 / 2020)$ 